Methods Data were collected at one London acute care Trust during two phases (June-August and September-November 2009) of the pandemic. Initial 24-h Acute Physiology and Chronic Health Evaluation (APACHE)-II scores and radiological findings were evaluated. All data were analysed by logistic regression (STATA)

Results 64 patients (31 female, 33 male; median age 32) hospitalised with H1N1/2009 were studied, comprising 22 white British, 19 African-Caribbean, 21 Asian and 2 of other ethnicity. Only three patients were aged $>60$ and only two deaths were encountered. Six pregnant patients were treated, including five in phase 2. Higher mean APACHE-II scores (7.9 vs 6.6) were recorded during this phase but were unrelated to demographic factors. ICU admission rate was high (12/64 patients, 19\%; including three who were pregnant). Asthma was the commonest co-morbidity (17/64 patients, 27\%) but only featured in two ICU cases. By contrast, pregnancy was a predictor of ICU admission (Pearson $\chi^{2} 4.2, \mathrm{P}$ $0.03)$. Asian males more than any other demographic were also more frequently identified than expected for this population $\left(\chi^{2}\right.$ 4.94, $\mathrm{p}=0.026$ ). Radiographic abnormalities were noted in $\geq 2$ quadrants of 28 (44\%) initial chest films, most frequently basal peribronchial thickening and perihilar opacities. Progressive infiltrates or multifocal consolidation were noted in 15/58 (26\%) patients, two-thirds $(9 / 15)$ of whom ultimately requiring intubation. Nosocomial infections or co-pathogens were found in six cases, all with underlying malignancy, transplanted organ or pregnancy.

Conclusions Most patients hospitalised with H1N1/2009 were young, with mild clinical and radiological disease. Asian males were more likely to be hospitalised with the illness, while pregnancy was correlated with an increased likelihood of ICU admission. Neither subgroup had evidence of worse acute illness severity. Basal nonlobar changes were the commonest radiographic finding. Overall, diffuse early abnormalities frequently preceded the development of severe and progressive pneumonia.

\section{P246 SUSPICION-PROMPTED TESTING REVEALS MISSED OPPORTUNITIES FOR DIAGNOSING NEW HIV CASES AMONG PATIENTS WITH COMMUNITY-ACQUIRED PNEUMONIA}

doi:10.1136/thx.2010.151068.47

S Safavi, F J K Chua, P Reilly, G K Russell. St Georges Hospital, London, UK

Introduction National UK policy for HIV testing ${ }^{1}$ recommends offering an HIV test to all patients with HIV indicator diseases such as tuberculosis (TB), Pneumocystis pneumonia, aspergillosis and bacterial pneumonia. Universal testing is advocated for all adults registering with a GP and all general medical hospital admissions in regions where HIV prevalence is $>2 / 1000$ population. Although 'opt out' HIV testing is a standard practice for patients with $\mathrm{TB}$, testing falls far short in other clinical settings.

Method A large number of community-acquired pneumonia (CAP) cases across three south London boroughs where HIV prevalence exceeds $2 / 1000^{2}$ are managed at our institution. All cases of CAP in 2008 (with one or more of: radiographic consolidation, CAP clinical case definition fulfilment, positive microbiology or pneumococcal urinary antigenaemia) were included in the present analysis. Demographic, HIV sampling and outcome data were collected.

Results 618 patients ( 325 or $53 \%$ male, 293 female) with a diagnosis of CAP were identified. The majority $(500,81 \%)$ were aged $>50$. Of the total, only 23 (3.7\%) patients underwent HIV testing, of whom 1 in 4 $(26 \%)$ tested positive. Half represented new diagnoses while the remainder had prior documentation of HIV positivity. Overall, 9/60 $(15 \%)$ of ethnically black (African-Caribbean) patients were tested, compared to only 6/429 (1.4\%) of White British patients. The rate of testing varied between age groups: $16 \%(19 / 118)$ of those aged $15-50$ were tested, in contrast to only $0.8 \%(4 / 500)$ of patients aged over 50 . Information regarding patients were declined testing was not available. Discussion Of individuals diagnosed with CAP who were HIVtested, the proportion of 'incident' positive testing (13\%) is approximately 5 times higher than expected if a policy of universal testing was practised. HIV testing was more often performed on younger or black patients. Despite serving a high prevalence population, HIV testing at this and likely many other institutions is under-utilised by medical teams even for patients with an HIV indicator pathology. Management of CAP in secondary care represents an opportunity to prevent a delayed diagnosis of HIV. Barriers to HIV testing need to be identified to reduce HIV mortality associated with late diagnosis.

\section{REFERENCES}

1. UK National Guidelines for HIV Testing (http://www.bhiva.org).

2. HIV in the United Kingdom: 2009 report. Health Protection Agency published November 2009

\section{P247 HIGH LEVELS OF IRON IN THE SOIL ARE ASSOCIATED WITH AN INCREASED INCIDENCE OF BOVINE TUBERCULOSIS IN CATTLE}

doi:10.1136/thx.2010.151068.48

G V O'Donovan, H J Milburn. Department of Respiratory Medicine, Guy's and St Thomas' NHS Foundation Trust, London, UK

Background Numbers and proportion of cases of bovine tuberculosis (BTB) in cattle are increasing, largely in the west country and Wales with few if any cases in the south and north east. Work by DEFRA demonstrates that TB in cattle is mutifactorial, as in humans, making some herds at greater risk of $\mathrm{TB}$ than others. Micronutrients could be one of these factors. Deficiencies, but also excesses, may cause secondary immunodeficiency and infection related morbidity in man. Different soil types contain different quantities of these trace elements and this will be reflected in pasture, hay and silage.

Objectives To determine whether certain rock and soil types predominate in areas with high incidence of BTB, and whether there is a case for investigating micronutrients in cattle.

Methods Information on cases of BTB and latest mapping of cases was provided by the Veterinary Research Laboratories (UK). Using information from the British Geological Survey and geological mapping, rock and soil types and their mineral contents were compared with density of cases of BTB.

Results There is a significant difference in the proportion of confirmed new incidents of BTB in the west of England $(6.8 / 100$ herds) and all other areas $(\mathrm{p}<0.001)$, and Wales $(3.2 / 100)$ and the north $(0.8 / 100)$ and east $(0.3 / 100)$ of England $(p<0.001)$. The rock types present in the west of England and Wales are rich in iron and aluminium while those in areas largely free of BTB are rich in calcium salts and oxides of silicon. Soils reflect the mineral content of the underlying rocks.

Discussion Iron deposits are common in areas with high numbers of cases of BTB, affecting quantities in pasture, hay, silage, and earthworms, a staple component of the diet of badgers, who are frequently blamed for this disease. Excess iron is associated with increased susceptibility to TB and more aggressive disease in man, and mycobacteria responsible for BTB need iron for their survival within the host.

Conclusions There is an association between BTB and high levels of iron in the soil. Further work is needed to determine levels of iron in affected cattle and its effect on immune responses. 